\title{
КЛИНИЧЕСКИЙ СЛУЧАЙ РАЗВИТИЯ АМИОДАРОН-ИНДУЦИРОВАННОГО ТИРЕОТОКСИКОЗА В ДЕТСКОМ ВОЗРАСТЕ
}

\author{
Т.Е. Иванникова, А.Л. Калинин, Н.А. Стребкова, В.А. Петеркова \\ ФГБУ «Национальный медицинский исследовательский центр эндокринологии» Минздрава \\ России, Москва
}

ВВЕДЕНИЕ: Амиодарон обычно используется для лечения рефрактерных предсердных или желудочковых аритмий. Примерно в 20\% случаев длительная терапия амиодароном может вызывать у пациентов субклинический или клинический гипотиреоз или гипертиреоз. Высокое содержание йода, содержащееся в амиодароне, нарушает ауторегуляцию щитовидной железы. Считается, что в патогенезе амиодарон-индуцированного тиреотоксикоза (АТ) участвуют два основных механизма. АТ 1 типа чаще встречается у пациентов с диффузным токсическим зобом или узловым зобом. Йод, высвобождаемый из препарата, приводит к повышению синтеза тиреоидных гормонов в существующих зонах автономии в железе. При АТ 2 типа наблюдается разрушение фолликулов щитовидной железы из-за токсичности амиодарона (подострый и деструктивный тиреоидит), что приводит к неконтролируемому выбросу гормонов щитовидной железы. Также описаны смешанные формы.

Представляем клинический случай тиреотоксикоза, который развился через два года от начала терапии амиодароном.

ОПИСАНИЕ КЛИНИЧЕСКОГО СЛУЧАЯ: Пациентка 15 лет наблюдалась по поводу бессимптомной желудочковой экстрасистолии с 10 лет.

В возрасте 13 лет был имплантирован кардиовертер-дефибриллятор и начата терапия амиодароном с постепенным увеличением до 500 мг в сутки. Через 2 года приема амиодарона по результатам УзИ - объем щитовидной железы 23,6 см³. В гормональном профиле стойкий эутиреоз на фоне отрицательного титра антител к рецептору ТТГ.

Через 2,5 года после начала терапии амиодароном пациентка обратилась с жалобами на учащение пульса. При исследовании гормонального профиля отмечалось снижение уровня ТТГ ᄀ- 0,0001 ME/мл (N: 0,35-5,5 мкME/мл) и выраженное повышение уровня свободного тироксина (Т4св.) - 52,11 пмоль/л (N: 11,5-22,7 пмоль/л) и свободного трийодтиронина (ТЗсв.) - 7,35 пмоль/л (N: 3,5-6,5 пмоль/л). Начата терапия преднизолоном 30 мг в сутки. После консультации кардиолога, начата отмена амиодарона. Через две недели после начала терапии удалось добиться нормализации уровней Т4Св. - 18,49 пмоль/л (N: 10,1-17,9 пмоль/л) и ТЗсв. - 3,5 пмоль/л (N: 2,8-6,3 пмоль/л). Также по результатам УзИ щитовидной железы отмечено уменьшение объема до $14,2 \mathrm{~cm}^{3}$. Проводится постепенное снижение дозы преднизолона.

ВыВОД: Данный клинический случай демонстрирует возникновение редкой патологии щитовидной железы на фоне терапии амиодароном в детском возрасте. В связи с этим требуется тщательное наблюдение за состоянием щитовидной железы до, во время и после лечения амиодароном. АТ может значительно ухудшить течение заболеваний сердечно-сосудистой системы. Ранняя и правильная диагностика амиодарон-индуцированного тиреотоксикоза позволяет незамедлительно начать соответствующее лечение с учетом состояния сердечно-сосудистой системы пациента.

КЛЮЧЕВЫЕ СЛОВА: тиреотоксикоз; амиодарон; амиодарон-индуцированный тиреотоксикоз; диффузный токсический зоб. 